\title{
Overexpression of WNT5B promotes COLO 205 cell migration and invasion through the JNK signaling pathway
}

\author{
YING ZHANG, LIANJIE LIN, YU JIN, YAN LIN, YONG CAO and CHANGQING ZHENG \\ The Second Department of Gastroenterology, Shengjing Hospital of China Medical University, \\ Shenyang, Liaoning 110022, P.R. China
}

Received December 8, 2015; Accepted January 8, 2016

DOI: $10.3892 / o r .2016 .4772$

\begin{abstract}
WNT5B is a member of the WNT family that has been reported to be overexpressed in a variety of cancer cell lines and tissues, including colorectal cancer (CRC). However, the potential roles of WNT5B in tumorigenesis have not been reported. In the present study, the WNT5B gene was transfected into CRC cells and generated a COLO 205 cell line with stable overexpression of WNT5B. MTT, wound healing and Transwell assays showed that overexpression of WNT5B significantly increased cell proliferation, migration and invasion capacities of the COLO 205 cells in vitro. Meanwhile, western blotting demonstrated that cells with stable expression of WNT5B showed increased protein expression levels and activities of matrix metalloproteinase (MMP) 2 and MMP 9. In addition, we also observed activation of the WNT/JNK signaling pathway in WNT5B-overexpressing cells. Subsequently, c-jun NH2-terminal kinase (JNK) was knocked down by RNA interference in the WNT5B-overexpressing COLO 205 cells. Knockdown of JNK significantly reduced the migratory capacity of the COLO 205 cells and decreased protein expression levels and activities of MMP 2 and 9 in vitro. In conclusion, our findings suggest that WNT5B may play an important role in the tumorigenesis of CRC.
\end{abstract}

\section{Introduction}

Colorectal cancer (CRC) is the third most common cancer in men and the second in women worldwide, accounting for roughly 1.36 million new cases and 694,000 deaths per year [http://globocan.iarc.fr/Pages/fact_sheets_cancer. aspx (accessed December 8, 2015)]. Surgery combined with radiochemotherapy is the principle therapeutic strategy for $\mathrm{CRC}$. However, the recurrence of advanced CRC cannot be

Correspondence to: Dr Changqing Zheng, The Second Department of Gastroenterology, Shengjing Hospital of China Medical University, 39 Huaxiang Road, Shenyang, Liaoning 110022, P.R. China

E-mail: changq_zheng2468@126.com

Key words: WNT5B, colorectal cancer, c-Jun NH2-terminal kinase, invasion prevented by current treatment. Twenty-five percent of CRC patients present with distant metastases at the time of initial diagnosis, and up to $50 \%$ of patients will develop metastatic disease (1). Due to the poor prognosis of CRC, understanding the underlying mechanisms of the development and progression of CRC is highly critical.

Glycoprotein WNTs have important functions in carcinogenesis and embryogenesis $(2,3)$. WNT signals transduce through at least three distinct intracellular signaling pathways including the canonical $\mathrm{WNT} / \beta$-catenin pathway, the WNT/ $\mathrm{Ca}^{2+}$ pathway and the WNT/polarity pathway (4). The canonical pathway has been intensely studied. In this pathway, WNT1, 2, 3 and 8 bind to members of the Frizzled (Fzd) gene family and members of the LDL-receptor-related protein (LRP) family $(5,6)$ and inhibit the degradation of $\beta$-catenin. In the non-canonical pathway, WNT5 and WNT11 bind to Frizzled and stimulate the WNT/Ca ${ }^{2+}$ and WNT/c-jun NH2-terminal kinase (JNK) pathways $(7,8)$.

Wingless-type MMTV integration site family member 5B (WNT5B) was first cloned by Tetsuroh Saitoh and Masaru Katoh in 2001. The WNT5b gene is a homologue of WNT5a, and the WNT5B protein shows $80.2 \%$ total amino-acid identity to WNT5A (9). Studies have demonstrated that WNT5B is expressed in esophageal, panereatic and breast cancer and embryonal tumor cell lines (10), which indicate a relationship between WNT5B expression and the malignant phenotype of these cancers. In addition, WNT5B was found to be overexpressed in leiomyoma cells compared with that in normal myometrial smooth muscle cells (11), and WNT5B was shown to be involved in the migratory ability of oral squamous cell carcinoma cells (12). These data suggest that WNT5B may be associated with tumorigenesis and metastasis. Importantly, a recent study found that the minor allele of WNT5B rs2010851 $\mathrm{T}>\mathrm{G}$ was significantly associated with a shorter tumor recurrence (13). Together with a finding that WNT5B was high expressed in the colonic tissues from ulcerative colitis patients, we hypothesized that WNT5B may be involved in the development of CRC. A previous study demonstrated that suppression of WNT5B inhibited cell growth, migration and mammosphere formation in triple-negative breast cancer cells (14), whereas its role in the invasion and metastasis of $\mathrm{CRC}$ has not yet been reported.

In the present study, the role of WNT5B in the proliferation, migration and invasion was evaluated in the COLO 205 
cell line. In addition, we aimed to ascertain whether the tumorpromoting effect of WNT5B is mediated by JNK.

\section{Materials and methods}

Cell culture. Human colon cancer cell lines HT-29, SW620, LoVo, HCT 15 and COLO 205 were purchased from the Type Culture Collection of the Chinese Academy of Sciences Cell Bank (Shanghai, China). The HT-29 cells were cultured in McCOY's 5A medium (Sigma-Aldrich, St. Louis, MO, USA). The SW620 cells were cultured in L15 medium (Gibco Life Technologies, Carlsbad, CA, USA). The LoVo cells were cultured in F12K medium (Sigma-Aldrich). The HCT 15 cells were cultured in RPMI-1640 medium (Gibco). The COLO 205 cells were cultured in RPMI-1640 medium. All the media were supplemented with 10\% FBS (Hyclone, Logan, UT, USA) and $1 \%$ antibiotics (penicillin-streptomycin; Sigma-Aldrich). The cells were maintained at $37^{\circ} \mathrm{C}$ in a $5 \% \mathrm{CO}_{2}$ incubator.

Plasmid construction. The plasmid expressing WNT5B was obtained by cloning the full coding sequences for the wildtype into the vector pcDNA3.1 (Invitrogen). Briefly, the open-reading frame (ORF) of the human WNT5B gene was amplified by PCR using the following primers: WNT5B forward, 5'-TTAGGATCCATGCCCAGCCTGCTGCTGCT-3 and reverse, 5'-CTCGAATTCCTATTTACAGATGTACTG GTCCACG-3'. The 5' end of the upstream primer pair and the $3^{\prime}$ end of the downstream primer pair had restriction enzyme BglII cutting sites GGATCC, and SalI cutting sites GAATTC, respectively. PCR products were separated by electrophoresis using $1.0 \%$ polyacrylamide gels, and the target fragment was purified and isolated using the Agarose Gel DNA Recovery kit (BioTeke Corporation, Beijing, China). The purified PCR fragment was ligated into the BamHI/XhoI (Takara Bio, Dalian, China) digested pcDNA3.1 vector to yield the pcDNA3.1-WNT5B construct. The code sequence of the plasmid was confirmed by sequencing.

Stable transfection. COLO 205 cells were seeded into a 24-well plate at a density of $1 \times 10^{5}$ cells/well without antibiotics. Cells were transfected with the pcDNA3.1-WNT5B plasmid using Lipofectamine 2000 (Invitrogen) and selected with $600 \mu \mathrm{g} / \mathrm{ml} \mathrm{G} 418$ (Invitrogen) for 2 weeks. Subsequently, individual colonies were isolated, expanded and maintained in $300 \mu \mathrm{g} / \mathrm{ml} \mathrm{G} 418$. The overexpression of WNT5B in these clones was confirmed by quantitative real-time PCR and western blotting.

Transfection with small interfering RNA (siRNA) targeting $J N K$. JNK siRNA and scramble siRNA were purchased from Genechem Co.,Ltd.(Shanghai, China). The siRNA sequence for JNK targeting was 5'-GCCCAGUAAUAUAGUAGUATT-3'. Scramble siRNA consisted of a scrambled sequence that does not lead to the specific degradation of any known cellular mRNA. siRNA transient transfection was performed using Lipofectamine 2000 (Invitrogen) according to the recommended instructions.

MTT assay. Cell proliferation was analyzed in vitro using the tetrazolium salt 3-(4,5-dimethylthiazol-2-yl)-2,5-diphe- nyltetrazolium bromide (MTT) method. Cells were seeded into 96 -well plates at the density of $5 \times 10^{3} /$ well. MTT solution (100 $\mu 1,0.5 \mathrm{mg} / \mathrm{ml}$; Sigma-Aldrich) was added into each well and incubated for $4 \mathrm{~h}$ at $37^{\circ} \mathrm{C}$. The supernatant was then removed, and the resultant formazan crystals were dissolved in dimethyl sulfoxide (DMSO; Sigma-Aldrich). The absorbance value was read at $570 \mathrm{~nm}$ using a microplate reader.

Wound healing assay. Cell mobility was assessed by a wound healing assay in vitro. Approximately $5 \times 10^{5}$ cells were seeded into 6-well plates until confluent. An incision was made in the central area with a $200-\mu 1$ pipette tip. After being washed twice with serum-free medium, the cells were then allowed to migrate into the cell-free area. The cells were photographed at 0 and $24 \mathrm{~h}$ using a microscope (Motic China Group Co., Ltd., Xiamen, China) at a magnification of $\mathrm{x} 100$. Cell migration was calculated as the mean percentage of the cell migrated distance compared with the initial wound distance. The experiment was performed in triplicate with three independent repeats.

In vitro Matrigel invasion assay. Twenty-four-well Transwell chambers (Costar, Cambridge, MA, USA) containing polycarbonate filters with $8-\mu \mathrm{m}$ pores coated with Matrigel $(1 \mathrm{mg} / \mathrm{ml}$, BD Biosciences, San Jose, CA, USA) were used for cell invasion capacity measurement in vitro. Approximately $5 \times 10^{4}$ cells in $500 \mu \mathrm{l}$ of serum-free medium were seeded into the upper chamber. Medium (750 $\mu \mathrm{l})$ containing $10 \%$ FBS was added to the lower chamber to attract cells. After allowing the cells to invade for $24 \mathrm{~h}$, the non-invasive cells were removed. The cells that penetrated the lower surface of the membrane were fixed in methanol and stained with hematoxylin. The numbers of the invasive cells were determined from six random fields using a microscope (Motic China Group Co., Ltd.) at a magnification of $\mathrm{x} 200$.

RNA extraction and RT-PCR. Total RNA was extracted from the cells using the RNAsimple Total RNA kit [Tiangen Biotech (Beijing) Co., Ltd, Beijing, China] according to the manufacturer's introductions. cDNA was synthesized using Super Moloney Murine Leukemia Virus Reverse Transcriptase (BioTeke Corp., Beijing, China). cDNA was then amplified by SYBR-Green (Solarbio) based real-time PCR on an Exicycler $^{\mathrm{TM}} 96$ thermal block (Bioneer, Daejeon, Korea). The primers used were as follows: WNT5B forward primer, 5'-CGTGGAGTACGGCTACCGCT-3' and reverse primer, 5'-CAGGCTACGTCTGCCATCTTAT-3'; JNK forward primer, 5'-GGATATAGCTTTGAGAAACTCTTCC-3', and reverse primer, TCTAACTGCTTGTCAGGGATCTT-3'; $\beta$-actin forward primer, 5'-CTTAGTTGCGTTACACCCTTT CTTG-3' and reverse primer, 5'-CTGTCACCTTCACCGTT CCAGTTT-3'. Relative mRNA expression normalized to $\beta$-actin was calculated by $2^{-\Delta \mathrm{Ct}}$. The $2^{-\Delta \Delta \mathrm{Ct}}$ method was used for fold change calculation.

Western blotting. The cells were dissolved in NP-40 lysis buffer (Beyotime) containing 1\% Triton X-100 with $1 \mathrm{mM}$ phenylmethanesulfonyl fluoride and quantified using a commercial BCA protein assay kit (Beyotime). Samples containing $40 \mu \mathrm{g}$ proteins were heat-denatured and separated using sodium dodecyl sulfate polyacrylamide 
gel electrophoresis (SDS-PAGE), and then transferred onto polyvinylidene fluoride membranes (EDM Millipore, Billerica, MA, USA). After a block stage using 5\% non-fat milk, the membranes were incubated with WNT5B $(1: 200$, sc-109464; Santa Cruz Biotechnology, Inc., Dallas, TX, USA), MMP 2 (1:400, BA0569) and MMP 9 (1:400, BA05730 (both from Boster, Wuhan, China), c-jun (1:500, bs-0670R), p-c-jun (1:500, bs-3209R), JNK (1:500, bs-10562R) and p-JNK (1:500, bs-1640R) (all from Bioss, Beijing, China) primary anibodies at $4^{\circ} \mathrm{C}$ overnight. Subsequently, the membranes were washed twice using TBST and incubated with goat anti-rabbit immunoglobulin G (IgG)-HRP-conjugated secondary antibodies (1:5000, Beyotime) for $45 \mathrm{~min}$ at $37^{\circ} \mathrm{C}$. The blots were visualized using enhanced chemiluminescence regent (Wanleibio) on X-ray film (Fuji Photo Film Co., Ltd., Tokyo, Japan). The protein levels were quantified by gray analysis using Gel-Pro-Analyzer software (Media Cybernetics, Bethesda, MD, USA). The grey levels are expressed as a percentage of $\beta$-actin levels (loading control).

Gelatin zymography. Activities of MMP 2 and MMP 9 in the culture medium of cells were analyzed using gelatin zymography as previously reported (15). The medium was collected and centrifuged at 1,000 $\mathrm{xg}$ for $2 \mathrm{~min}$ to remove debris. Equal amounts of protein were separated on a $10 \%$ SDS-PAGE gel containing $1 \mathrm{mg} / \mathrm{ml}$ gelatin. The gels were washed in renaturing buffer $[50 \mathrm{mmol} / 1$ Tris ( $\mathrm{pH} 7.6), 5 \mathrm{mmol} / 1 \mathrm{CaCl}_{2}, 1 \mu \mathrm{mol} / 1 \mathrm{ZnCl}_{2}$ and $2.5 \%$ Triton $\mathrm{X}-100$ ] twice and then incubated overnight in developing buffer [50 mmol/1 Tris- $\mathrm{HCl}$ (pH 7.6), $200 \mathrm{mmol} / 1$ $\mathrm{NaCl}, 5 \mathrm{mmol} / 1 \mathrm{CaCl}_{2}, 1 \mu \mathrm{mol} / 1 \mathrm{ZnCl}_{2}$ and $\left.0.02 \% \mathrm{Brij}\right]$ at $37^{\circ} \mathrm{C}$ for $40 \mathrm{~h}$. The gels were then stained using $0.05 \%$ Coomassie blue R-250 in 30\% methanol and 5\% acetic acid and destained with destaining solution A (30\% methanol, $10 \%$ acetic acid) for $0.5 \mathrm{~h}, \mathrm{~B}$ (20\% methanol, $10 \%$ acetic acid) for $1 \mathrm{~h}$, and $\mathrm{C}$ (10\% methanol, $5 \%$ acetic acid) for $2 \mathrm{~h}$. The image was captured and analyzed using gel imaging and analysis system (WD-9413B; Beijing Liuyi Biotechnology Co., Ltd., Beijing, China)

Immunofluorescence. COLO 205 cells were cultured on glass coverslips and fixed with $4 \%$ paraformaldehyde (Sinopharm Chemical Reagent Co., Ltd, Beijing, China) for $15 \mathrm{~min}$. The cells were then treated with $0.5 \%$ Triton X-100 for $10 \mathrm{~min}$ and blocked with goat serum (Solarbio Science \& Technology, Co., Ltd., Beijing, China) for $15 \mathrm{~min}$ at room temperature. Subsequently, the cells were incubated with anti-p-c-jun primary antibody (1:200, bs-3209R; Bioss) at $4^{\circ} \mathrm{C}$ overnight. After a washing stage with $1 \mathrm{X}$ PBS, the cells were incubated in secondary antibody coupled to Cy3 (1:200; Beyotime) in darkness for $1 \mathrm{~h}$ at room temperature. The cells were then counterstained with 4',6-diamidino-2-phenylindole (DAPI) (Biosharp, Hefei, China) and observed using a fluorescence microscope (BX53; Olympus, Tokyo, Japan).

Statistical analysis. Data are presented as the mean \pm SD. The results were assessed using a one-way analysis of variance (ANOVA) followed by an LSD test for comparisons among groups. Statistical analyses were conducted using SPSS 19 (IBM SPSS, Armonk, NY, USA). P-values <0.05 were considered to be indicative of statistical significance.

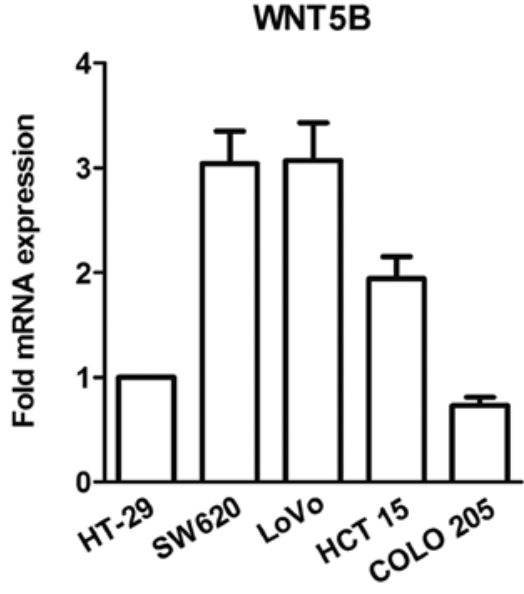

Figure 1. WNT5B mRNA levels were detected by real-time RT-PCR in human colon cancer cell lines. $\beta$-actin was used as an endogenous control. Results are presented as mean \pm SD of three independent experiments.

\section{Results}

Effect of WNT5B on cell proliferation, migration and invasion. Prior to the studies, WNT5B expression in five cell lines was tested using real-time PCR. COLO 205 cells, which exhibited the lowest level of WNT5B expression (Fig. 1), were used in the following studies.

Expression of WNT5B mRNA in COLO 205 cells transfected with the pcDNA3.1-WNT5B or empty vector is shown in Fig. 2A. Six colons were chosen randomly to be examined. WNT5B mRNA expression was significantly increased in the six colons compared to the untreated COLO 205 cells or cells transfected with the empty vector (mock). Colon 2, the lowest one (WNT5B-L), and colon 6, the highest one (WNT5B-H), were used in the following experiments.

The proliferation of cells was assessed using MTT assay. As shown in Fig. 2B, at 12, 24 and 48 h, cell proliferation was significantly increased in the WNT5B-H cells. A modest increase in cell proliferation was also found in the WNT5B-L cells, but did not achieve a significant difference. Cell migration and invasion were assessed using wound healing and Transwell assays. Migration and invasion activities were also significantly increased in the WNT5B-H cells compared to the mock, although the WNT5B-L cells did not show significant changes (Fig. 2C and D).

Effects of WNT5B on MMP 2 and MMP 9 expression and secretory activities. The protein expression of MMP 2 and MMP 9 was examined using western blotting. Secretory activities of MMP 2 and MMP 9 were examined using gelatin zymography. As illustrated in Fig. 3, the protein expression and secretory activities were significantly enhanced by WNT5B transfection in a WNT5B expression-dependent manner.

Effects of WNT5B on activity of the WNT/JNK signaling pathway. The activation of the WNT/JNK signaling pathway was evaluated using western blotting and immunofluorescence analysis. As shown in the Fig. 4A and B, WNT5B overexpression markedly induced phosphorylation of JNK and c-jun in 
A

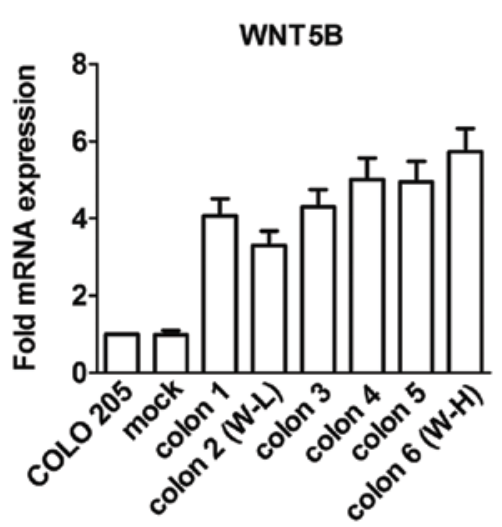

B

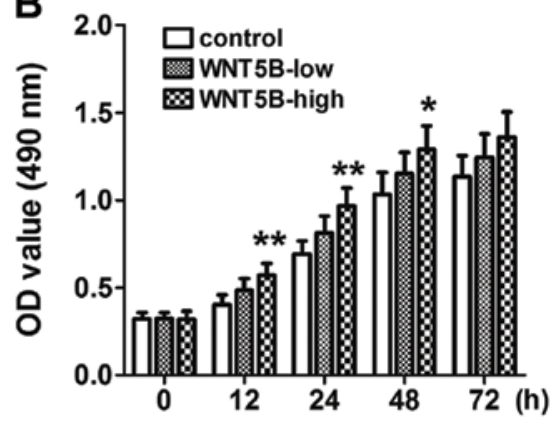

C

control

WNT5B-L
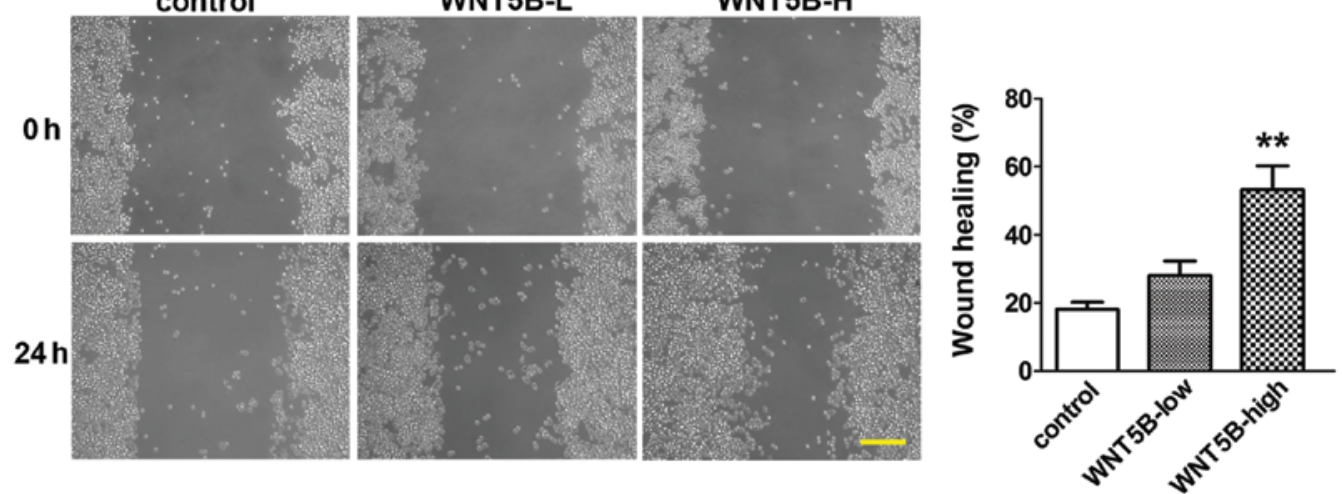

D
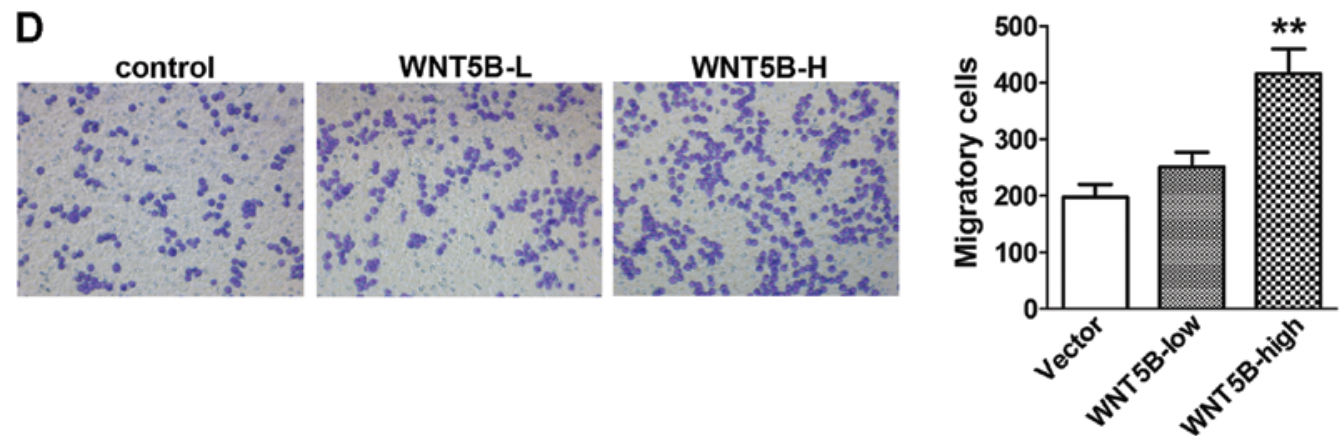

Figure 2. Effect of WNT5B on the proliferation, migration and invasion of colon cancer COLO 205 cells. (A) Real-time PCR analysis of WNT5B mRNA expression in COLO 205 cells stably transfected with the WNT5B gene. (B) Effect of WNT5B on the proliferation of COLO 205 cells. Cells in the WNT5Bhigh group showed a significant increase in cell proliferation in comparison with the cells in the vector group at 12, 24 and $48 \mathrm{~h}$ after being seeded. (C and D) Effect of WNT5B on the migration and invasion of COLO 205 cells. Cells in the WNT5B-high group demonstrated a markedly increase in the abilities of migration and invasion. Results are presented as mean \pm SD of three independent experiments. ${ }^{*} \mathrm{P}<0.05$ and ${ }^{* *} \mathrm{P}<0.01$ vs. the vector group. Scale bar, $100 \mu \mathrm{m}$.

the COLO 205 cells, and the phospho-protein/total protein ratios in the WNT5B-high group were higher than that in the WNT5B-low group. The immunofluorescence analysis confirmed the findings of the western blot analysis (Fig. 4C). The WNT5B-overexpressing cells showed significantly higher levels of c-jun phosphorylation than that noted in the control cells.

Effect of JNK siRNA transfer on the migration activity of the WNT5B transfectants. To evaluate the involvement of JNK in the increased migration activities of the WNT5Btransfected COLO 205 cells, JNK siRNA was tranfected into the WNT5B-H cells. As illustrated in Fig. 5A and B, the expression levels of JNK mRNA and protein were markedly decreased by RNA interference of JNK. Migration activity of the JNK siRNA-transfected WNT5B-H cells was markedly decreased in comparison with the mock cells (Fig. 5C).

Effect of JNK siRNA transfer on MMP 2 and MMP 9 expression and secretory activities of WNT5B transfectants. As shown in Fig. 6, JNK siRNA transfection significantly inhibited the secretory activities and protein expression levels of MMP 2 and MMP 9.

\section{Discussion}

Our present findings showed, for the first time, that WNT5B promoted cell proliferation, migration and invasion abilities in 
A
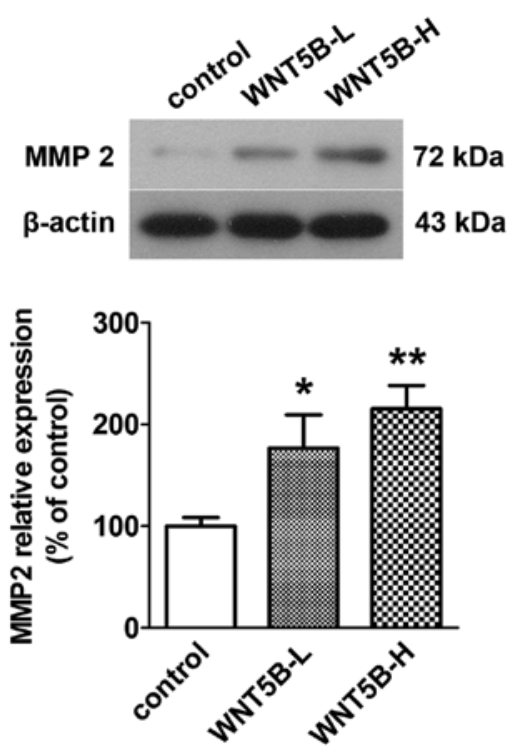

C

D

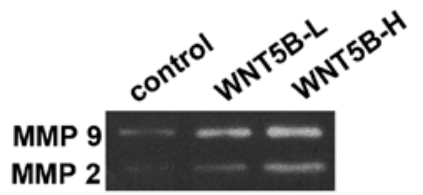

B
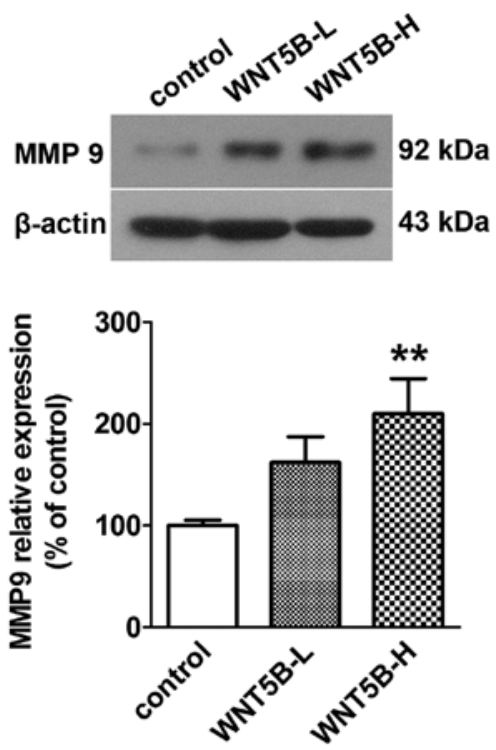

E

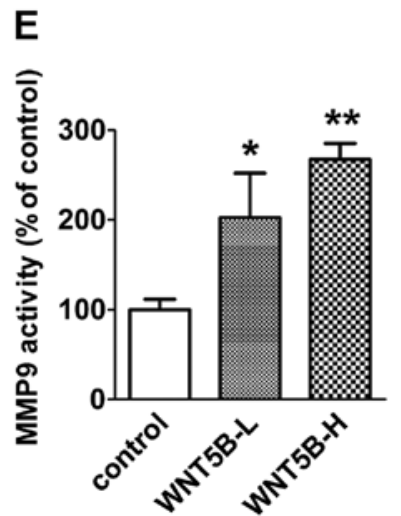

Figure 3. Effect of WNT5B overexpression on MMP 2 and 9 protein expression and secretory activities. (A and B) Western blotting of WNT5B overexpression on the protein expression of MMP 2 and 9 in the COLO 205 cells. MMP 2 and 9 were upregulated in the WNT5B-overexpressing cells. (C-E) Gelatin zymography analysis of WNT5B overexpression on the activities of MMP 2 and 9 in COLO 205 cells. The secretory activities of MMP 2 and 9 were increased in the WNT5B-overexpressing cells. Results are presented as mean $\pm \mathrm{SD}$ of three independent experiments. ${ }^{*} \mathrm{P}<0.05$ and ${ }^{* *} \mathrm{P}<0.01$ vs. the vector group.

human colon cancer cell line COLO 205 in vitro through the JNK signaling pathway.

Migration and invasion are important prerequisites of cancer progression and metastasis. In the present study, wound healing and Transwell invasion assays were conducted to determine the effects of WNT5B overexpression on the migration and invasion abilities of COLO 205 cells. The results showed that overexpression of WNT5B significantly increased the invasive and metastatic ability of the COLO 205 cells in vitro. Matrix metalloproteinases (MMPs), a family of zinc-dependent endopeptidases $(16,17)$, play an important role in proteolytic degradation of the basement membrane and extracellular matrix (ECM), which is the initial step of tumor invasion and metastasis. In particular, MMP 2 and MMP 9 have been considered to be associated with increased invasiveness in various cancer cells (18). Invadopodia, actin-rich, finger-like cellular membrane projections located at the ventral side of the cell, are involved in ECM degradation through disintegrating the basement membrane (19). MMP 2 and MMP 9 have been shown to be enriched in invadopodia where they contribute to ECM degradation in vitro and in vivo (19). The present study demonstrated a marked increase in protein levels and secretory activities of MMP 2 and MMP 9 in the COLO 205 cells with WNT5B overexpression. Although the present data do not provide direct evidence for the effect of WNT5B on MMPs, overexpression of WNT5B may promote the metastatic ability of COLO 205 cells by upregulating MMP 2 and MMP 9 expression and increasing their secretory activities.

$\mathrm{JNK}$ is a member of the mitogen activated protein kinases (MAPK) and is involved in non-canonical WNT signaling (20). In the present study, we examined the expression and phosphorylation of JNK and c-jun. The results revealed that the phosphorylation levels of the two proteins were increased in the WNT5B transfectants compared to the mock cells, and siRNA-mediated gene knockdown of JNK in the WNT5B transfectants was shown to decrease their migratory activity. This suggests that WNT5B may regulate cell migration activity at least partly through regulation of the WNT/JNK signaling pathway in COLO 205 cells.

The role of JNK in tumorigenesis is ambiguous. On the one hand, accumulating evidence suggests that JNK has tumor-promoting function. Mice with JNK1 gene double knockout exhibited a marked decrease in the development of $\mathrm{N}$-methyl-N-nitrosourea-induced gastric tumors compared 

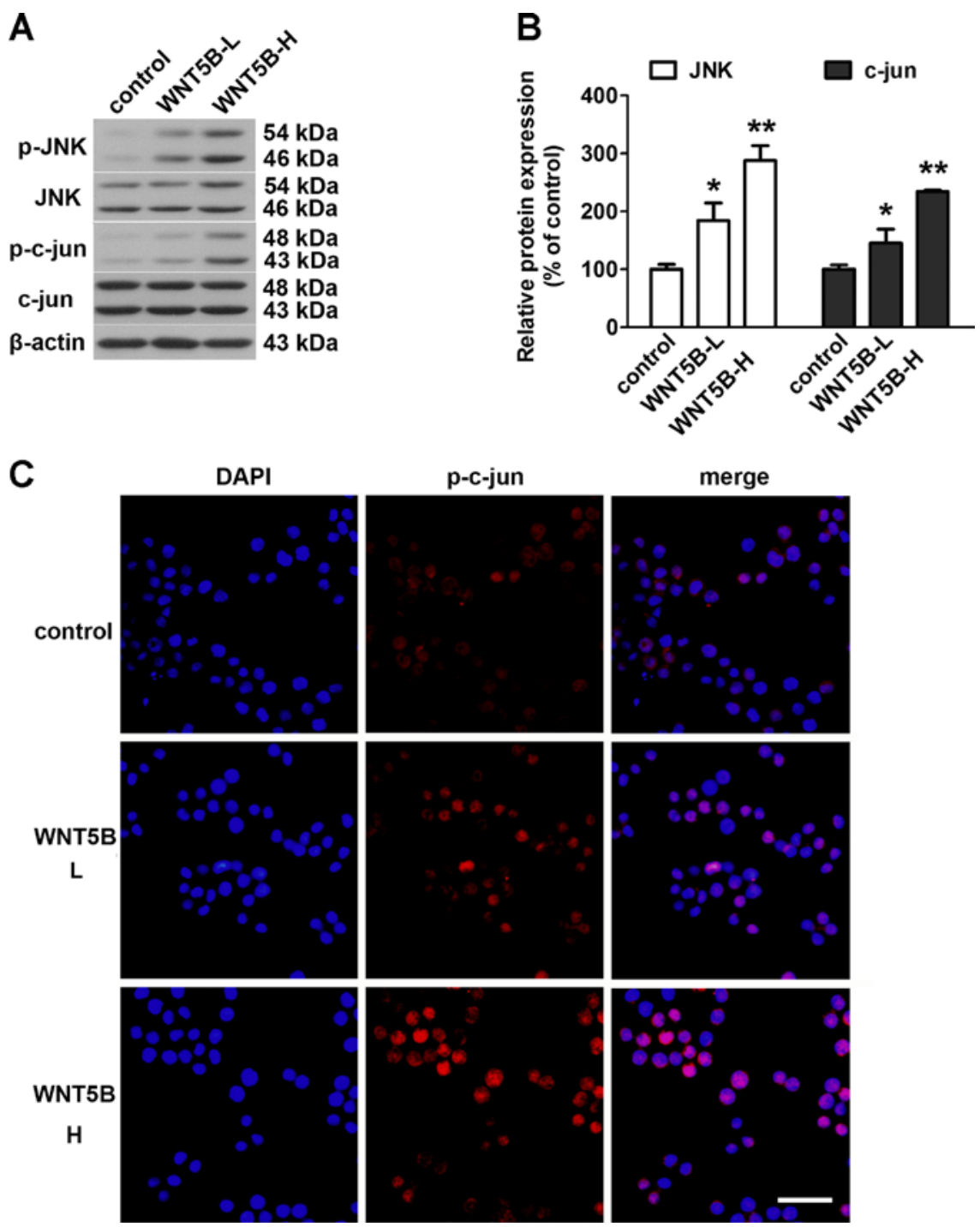

Figure 4. Effect of WNT5B overexpression on JNK signaling. (A and B) Effect of WNT5B overexpression on c-jun and JNK protein expression as determined by western blot analysis. WNT5B overexpression increased the phosphorylation of these two proteins. Results are presented as mean \pm SD of three independent experiments. ${ }^{*}<0.05$ and ${ }^{* *} \mathrm{P}<0.01$ vs. the vector group. (C) Immunofluorescence analysis of the effect of WNT5B overexpression on c-jun phosphorylation in COLO 205 cells. Phosphorylated c-jun expression was increased in the WNT5B-overexpressing cells. Scale bar, $50 \mu \mathrm{m}$.

with wild-type mice (21). Moreover, JNK1/- mice were shown to be much less susceptible to hepatocarcinogenesis caused by diethylnitrosamine, and the absence of JNK1 resulted in reduced cell proliferation and tumor neovascularization (22). In addition, activation of JNK signaling has been found to accelerate colitis-induced tumorigenesis (23). On the other hand, other findings indicate that JNK has tumor-suppressing function. She and colleagues demonstrated that $\mathrm{JNK} 1^{-1-}$ mice were more susceptible to TPA-induced skin tumor development than wild-type mice (24). Mice lacking the JNK1 gene were found to exhibit increased intestinal tumor formation (25). Data from WNT family member-associated studies showed discrepancies as well. WNT5A, the homologous gene of WNT5B, has been demonstrated to enhance the motility of malignant cells and tumor invasion in various types of cancer $(26,27)$. It was found to regulate cell migration through JNK activation in pancreatic cancer and human osteosarcoma cells $(28,29)$, which suggests that the pro-tumorigenic function of WNT5A is associated with JNK activation. WNT11 gene overexpression was found to induce JNK and c-jun phosphory- lation and increase migration and invasion in CRC HCT-116 cells (30), and downregulation of Frizzled-7 with siRNA resulted in decreased phosphorylation of JNK and migration in HCT-116 cells (31), which indicate the involvement of the WNT/JNK signaling pathway in the tumor-promoting activity of WNT11/Frizzled-7. However, WNT7a/ Frizzled-9 signaling was found to inhibit the growth of non-small cell lung cancer cells through activation of the JNK pathway (32). In the present study, similar to its homologous protein WNT5A, WNT5B promoted the migration and invasion through activation of the JNK signaling pathway in COLO 205 cells, and increased MMP 2 and MMP 9 expression and secretory activities as well. This discrepancy can be attributed to the difference in cell type, organ or stimuli, while the detailed mechanisms require further investigation.

In conclusion, this is the first study to show that WNT5B may play an important role in the regulation of migration and invasion activities of CRC cells through the WNT/JNK signaling pathway. These results suggest that WNT5B/JNK may be involved in CRC progression. 

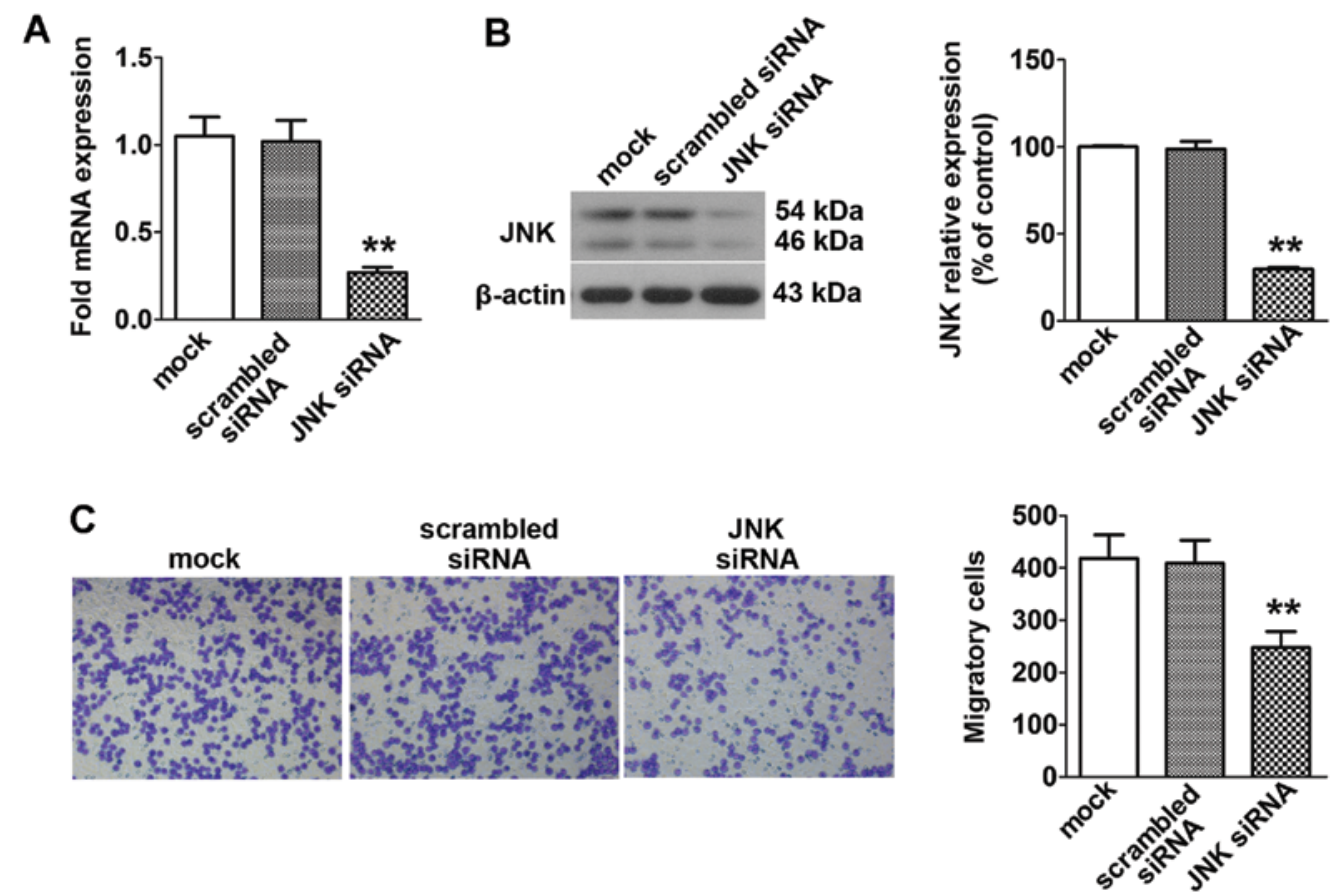

Figure 5. Effect of JNK knockout on cell invasion in the WNT5B transfectants. (A and B) Real-time PCR and western blot analysis of JNK expression following siRNA-mediated knockdown of JNK. The expression of JNK was diminished in the WNT5B transfectant after JNK-siRNA treatment. (C) JNK knockdown significantly decreased the invasive ability of the COLO 205 cells transfected with the WNT5B gene. Results are presented as mean \pm SD of three independent experiments. ${ }^{* *} \mathrm{P}<0.01$ vs. the mock group.

A
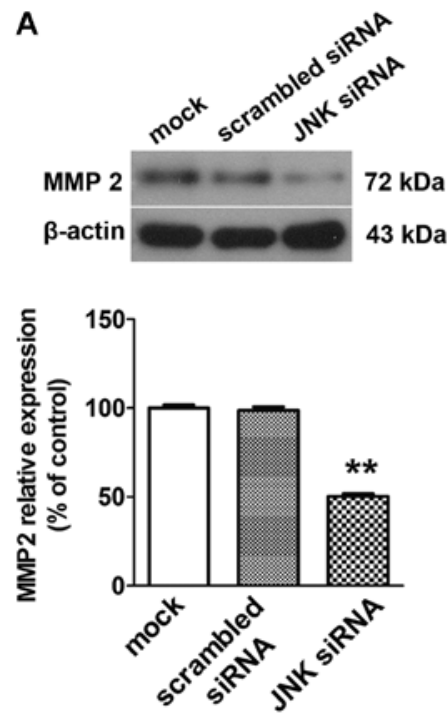

C

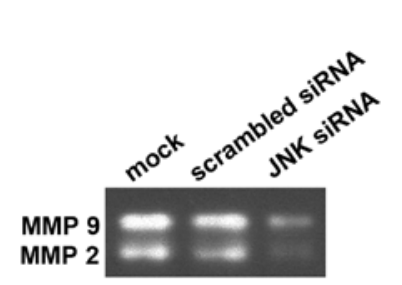

D

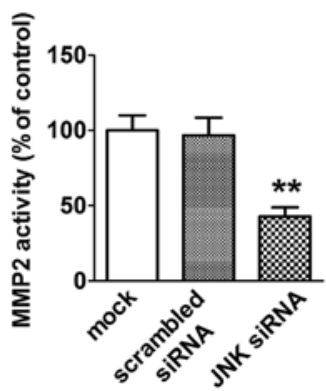

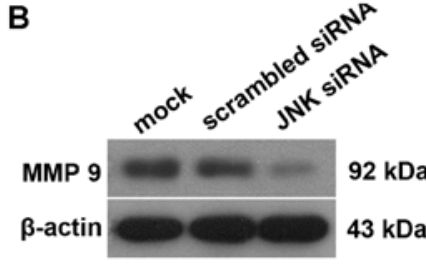

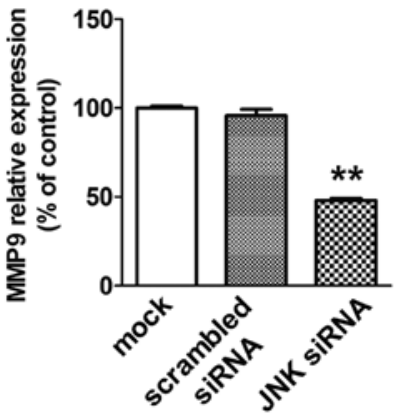

$\mathrm{E}$

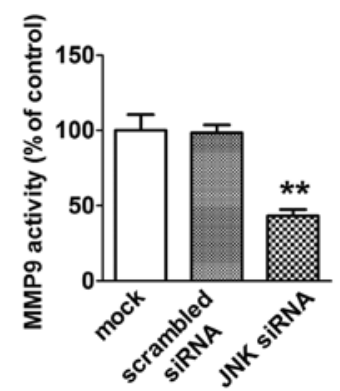

Figure 6. Effect of JNK knockdown on the expression and secretory activities of MMP 2 and 9 in the WNT5B transfectant. (A and B) JNK knockdown significantly decreased protein expression of MMP 2 and MMP 9 in the WNT5B-transfected COLO 205 cells. (C-E), JNK knockdown significantly decreased the secretory activities of MMP 2 and MMP 9 in the WNT5B-transfected COLO 205 cells. Results are presented as the mean \pm SD of three independent experiments. ${ }^{* *} \mathrm{P}<0.01$ vs. the mock group. 


\section{References}

1. Worni M, Shah KN and Clary BM: Colorectal cancer with potentially resectable hepatic metastases: optimizing treatment. Curr Oncol Rep 16: 407, 2014.

2. Moon RT, Brown JD and Torres M: WNTs modulate cell fate and behavior during vertebrate development. Trends Genet 13: 157-162, 1997.

3. Peifer M and Polakis P: Wnt signaling in oncogenesis and embryogenesis - a look outside the nucleus. Science 287: 1606-1609, 2000

4. Miller JR: The Wnts. Genome Biol 3: REVIEWS3001, 2002.

5. Bejsovec A: Wnt signaling: an embarrassment of receptors. Curr Biol 10: R919-R922, 2000.

6. Pandur P and Kühl M: An arrow for wingless to take-off. BioEssays 23: 207-210, 2001

7. Kühl M, Sheldahl LC, Park M, Miller JR and Moon RT: The $\mathrm{Wnt} / \mathrm{Ca}^{2+}$ pathway: a new vertebrate Wnt signaling pathway takes shape. Trends Genet 16: 279-283, 2000.

8. Weston CR and Davis RJ: The JNK signal transduction pathway. Curr Opin Genet Dev 12: 14-21, 2002.

9. Saitoh T and Katoh M: Molecular cloning and characterization of human WNT5B on chromosome 12p13.3 region. Int J Oncol 19: 347-351, 2001

10. Saitoh T and Katoh M: Expression and regulation of WNT5A and WNT5B in human cancer: up-regulation of WNT5A by TNFalpha in MKN45 cells and up-regulation of WNT5B by beta-estradiol in MCF-7 cells. Int J Mol Med 10: 345-349, 2002.

11. Mangioni S, Viganò $P$, Lattuada $D$, Abbiati $A$, Vignali $M$ and Di Blasio AM: Overexpression of the Wnt5b gene in leiomyoma cells: implications for a role of the Wnt signaling pathway in the uterine benign tumor. J Clin Endocrinol Metab 90: 5349-5355, 2005.

12. Takeshita A, Iwai S, Morita Y, Niki-Yonekawa A, Hamada M and Yura Y: Wnt5b promotes the cell motility essential for metastasis of oral squamous cell carcinoma through active $\mathrm{Cdc} 42$ and RhoA. Int J Oncol 44: 59-68, 2014.

13. Páez D, Gerger A, Zhang W, Yang D, Labonte MJ, Benhanim L, Kahn M, Lenz F, Lenz C, Ning Y, et al: Association of common gene variants in the $\mathrm{WNT} / \beta$-catenin pathway with colon cancer recurrence. Pharmacogenomics J 14: 142-150, 2014.

14. Yang L, Perez AA, Fujie S, Warden C, Li J, Wang Y, Yung B, Chen YR, Liu X, Zhang H, et al: Wnt modulates MCL1 to control cell survival in triple negative breast cancer. BMC Cancer 14: $124,2014$.

15. Huang Q, Shen HM and Ong CN: Inhibitory effect of emodin on tumor invasion through suppression of activator protein-1 and nuclear factor-kappaB. Biochem Pharmacol 68: 361-371, 2004.

16. Min KW, Kim DH, Do SI, Kim K, Lee HJ, Chae SW, Sohn JH Pyo JS, Oh YH, Kim WS, et al: Expression patterns of stromal MMP-2 and tumoural MMP-2 and -9 are significant prognostic factors in invasive ductal carcinoma of the breast. APMIS 122: 1196-1206, 2014.

17. Stellas D and Patsavoudi E: Inhibiting matrix metalloproteinases, an old story with new potentials for cancer treatment. Anticancer Agents Med Chem 12: 707-717, 2012.

18. Bauvois B: New facets of matrix metalloproteinases MMP-2 and MMP-9 as cell surface transducers: outside-in signaling and relationship to tumor progression. Biochim Biophys Acta 1825 29-36, 2012.
19. Murphy DA and Courtneidge SA: The 'ins' and 'outs' of podosomes and invadopodia: characteristics, formation and function. Nat Rev Mol Cell Biol 12: 413-426, 2011.

20. Veeman MT, Axelrod JD and Moon RT: A second canon. Functions and mechanisms of beta-catenin-independent Wnt signaling. Dev Cell 5: 367-377, 2003.

21. Shibata W, Maeda S, Hikiba Y, Yanai A, Sakamoto K, Nakagawa $H$, Ogura K, Karin $M$ and Omata $M$ : c-Jun $\mathrm{NH}_{2}$-terminal kinase 1 is a critical regulator for the development of gastric cancer in mice. Cancer Res 68: 5031-5039, 2008.

22. Sakurai T, Maeda S, Chang L and Karin M: Loss of hepatic NF-kappa B activity enhances chemical hepatocarcinogenesis through sustained c-Jun N-terminal kinase 1 activation. Proc Natl Acad Sci USA 103: 10544-10551, 2006.

23. Sancho R, Nateri AS, de Vinuesa AG, Aguilera C, Nye E, Spencer-Dene B and Behrens A: JNK signalling modulates intestinal homeostasis and tumourigenesis in mice. EMBO J 28: 1843-1854, 2009.

24. She QB, Chen N, Bode AM, Flavell RA and Dong Z: Deficiency of c-Jun-NH(2)-terminal kinase-1 in mice enhances skin tumor development by 12-O-tetradecanoylphorbol-13-acetate. Cancer Res 62: 1343-1348, 2002.

25. Tong C, Yin Z, Song Z, Dockendorff A, Huang C, Mariadason J, Flavell RA, Davis RJ, Augenlicht LH and Yang W: c-Jun $\mathrm{NH}_{2}$-terminal kinase 1 plays a critical role in intestinal homeostasis and tumor suppression. Am J Pathol 171: 297-303, 2007.

26. Kurayoshi M, Oue N, Yamamoto H, Kishida M, Inoue A, Asahara T, Yasui W and Kikuchi A: Expression of Wnt-5a is correlated with aggressiveness of gastric cancer by stimulating cell migration and invasion. Cancer Res 66: 10439-10448, 2006.

27. Yamanaka H, Moriguchi T, Masuyama N, Kusakabe M, Hanafusa H, Takada R, Takada S and Nishida E: JNK functions in the non-canonical Wnt pathway to regulate convergent extension movements in vertebrates. EMBO Rep 3: 69-75, 2002.

28. Nomachi A, Nishita M, Inaba D, Enomoto M, Hamasaki M and Minami Y: Receptor tyrosine kinase Ror2 mediates Wnt5a-induced polarized cell migration by activating c-Jun $\mathrm{N}$-terminal kinase via actin-binding protein filamin A. J Biol Chem 283: 27973-27981, 2008

29. Wei W, Li H, Li N, Sun H, Li Q and Shen X: WNT5A/JNK signaling regulates pancreatic cancer cells migration by phosphorylating paxillin. Pancreatology 13: 384-392, 2013.

30. Nishioka M, Ueno K, Hazama S, Okada T, Sakai K, Suehiro Y, Okayama N, Hirata H, Oka M, Imai K, et al: Possible involvement of WNT11 in colorectal cancer progression. Mol Carcinog 52: 207-217, 2013.

31. Ueno K, Hazama S, Mitomori S, Nishioka M, Suehiro Y, Hirata $H$, Oka M, Imai K, Dahiya R and HinodaY: Down-regulation of frizzled-7 expression decreases survival, invasion and metastatic capabilities of colon cancer cells. Br J Cancer 101: 1374-1381, 2009.

32. Winn RA, Marek L, Han SY, Rodriguez K, Rodriguez N, Hammond M, Van Scoyk M, Acosta H, Mirus J, Barry N, et al: Restoration of WNT-7a expression reverses non-small cell lung cancer cellular transformation through frizzled-9-mediated growth inhibition and promotion of cell differentiation. J Biol Chem 280: 19625-19634, 2005. 